\title{
Advances in the Analysis of Gunshot Residue and Other Trace Evidence using EDS and EBSD in the SEM
}

\author{
Frank Bauer ${ }^{1}$, Matthew Hiscock ${ }^{2}$ and Christian Lang $^{2}$ \\ 1. Oxford Instruments Nanoanalysis, Wiesbaden, Germany and Country. \\ 2. Oxford Instruments Nanoanalysis, High Wycombe, United Kingdom.
}

Scanning Electron Microscopy (SEM) based analysis is a standard tool in the analysis of trace evidence providing morphological and, in combination with EDS, also chemical information down to the sub micron scale. Many forensic science laboratories use SEM-EDS to analyse for gunshot residue (GSR) with samples taken from suspected shooters' hands, hairs and clothing. Other commonly analysed samples include broken glass, soil from suspects' shoes or paint. The aim of the analysis is to either conclusively link the evidence to a suspected source by matching chemical and morphological characteristics or to rule out such connections. In the case of GSR automated analysis is used to acquire morphological (from the electron image) and chemical (EDS) data from suspected GSR particles. Based on this data particles are then automatically classified according to ASTM 1588-10 ${ }^{\varepsilon 1}$ as either characteristic or consistent with GSR. This classification was relatively straight forward when most ammunition types used lead bearing primers which resulted in large populations of easy to detect, characteristic particles. However, with the advent of non-toxic ammunition, the particle populations typically detected have changed and fewer or no classically characteristic particles may be found in samples taken from shooters. Here we show that by adding data from EBSD analysis we can positively identify phases associated with the high temperatures and pressures associated with firing a handgun using non-toxic ammunition. Furthermore, we discuss how advances in both the software and hardware enable more accurate automated element identification at high speed which in turn allows us to link particle populations to the presence of a particular type of non-toxic ammunition which has so far only been achieved using principle component analysis of EDS spectra [1].

SINTOX $^{\circledR}$ is a commonly used primer in non-toxic ammunition that is based on tetrazene for sensitization, DDNP as an initial explosive and a pyro system consisting of Zinc peroxide and Titanium which replaces the lead styphnate in conventional ammunition. Its reaction products are particles containing $\mathrm{Zn}$ and $\mathrm{Ti}$ in varying concentration. However, most concentrations are fairly close to possible environmental sources and ASTM $1588-10^{\varepsilon 1}$ therefore classes them as consistent rather than characteristic. By combining the chemical data obtained from EDS analysis with crystallographic data from EBSD we could positively identify the presence of the $\mathrm{TiZn}_{2} \mathrm{O}_{4}$ spinel phase (figure 1). This phase is only formed at high temperatures and pressures and therefore is much less likely to be found environmentally, especially in structural paragenesis with $\mathrm{ZnO}$.

In order to answer the question of whether it is possible to identify a particular type of non-toxic ammunition by the type of primer used, we studied samples originating from shooters having fired known ammunition types. We also added several classes to the ASTM 1588-10 ${ }^{\varepsilon 1}$ classification scheme which were designed to pick out specific reaction compounds based on primer composition. When running the samples with the extended classification scheme, the new classes added clearly pick out the correct ammunition type (figure 2). While there are more non-toxic ammunition types on the market than we have identified here, we clearly show that by adding reaction products of different primers to the classification scheme we can distinguish between different types of ammunition which may add 
valuable information in cases where there is more than one shooter.

We can apply similar techniques to other types of trace evidence such as soil samples where we have previously shown [2] that we can successfully pick out certain mineral phases that are indicative of a certain geographical location. [3]

\section{References:}

[1] S. Hogg et al, J. Forensic Sci. (2015), p. 1556.

[2] R Morgan et al, EAFS Conference (2015), to be published.

[3] The authors thank the BKA Wiesbaden, Raggruppamento Carabinieri Investigazioni Scientifiche and all other laboratories who have provided samples for this study.
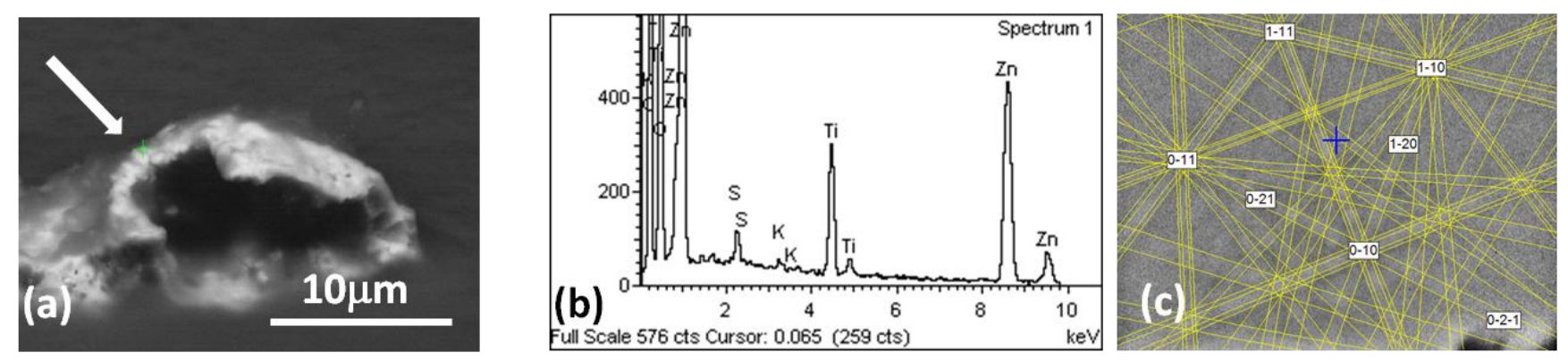

Figure 1. (a) electron image of a SINTOX particle with analysis point indicated by arrow. (b) EDS spectrum showing $\mathrm{O}$, Ti and $\mathrm{Zn}$ clearly identified. (c) EBSD pattern from the same location which has been indexed and shown to be consistent with the $\mathrm{TiZn}_{2} \mathrm{O}_{4}$ spinel phase.

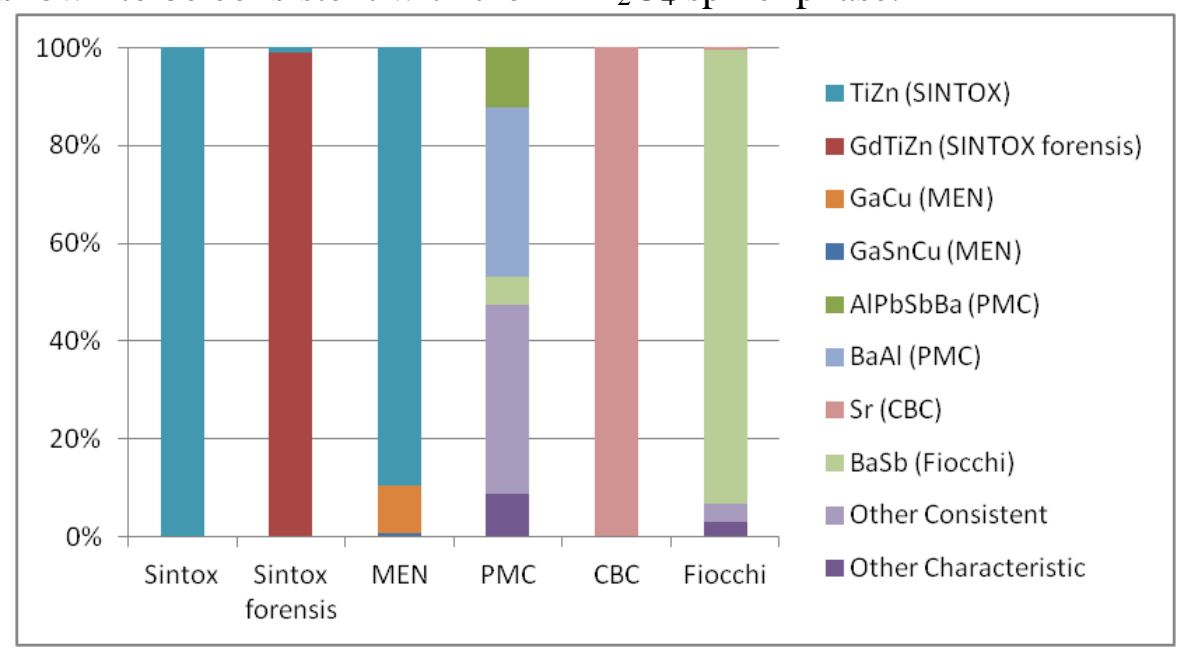

Figure 2. The graph shows the percentage of particles in the classes used to identify certain ammunition types for different samples. Each sample is labeled with the ammunition type used by the shooter. A good correspondence between the classes and the expected ammunition type is observed. 